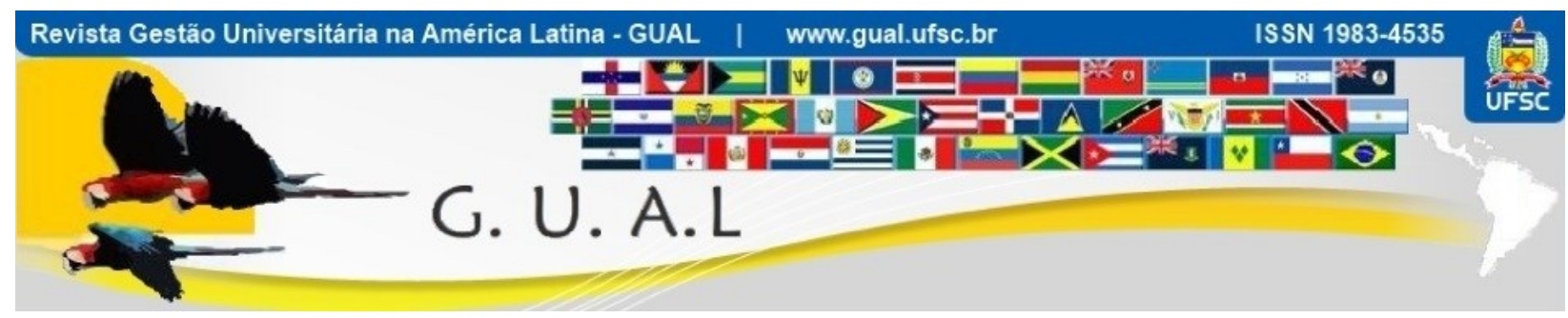

DOI: http://dx.doi.org/10.5007/1983-4535.2018v11n3p20

\title{
SATISFAÇÃO NO TRABALHO SOB A PERSPECTIVA DA ORIENTAÇÃO PARA O MERCADO INTERNO DE UMA INSTITUIÇÃO DE ENSINO PÚBLICA: DOCENTES $X$ ADMINISTRATIVO
}

\section{WORK SATISFACTION FROM THE PERSPECTIVE OF INTERNAL MARKET ORIENTATION IN A PUBLIC EDUCATION INSTITUTION: TEACHERS $X$ ADMINISTRATIVE STAFF}

\author{
Zamora Cristina dos Santos, Mestre \\ Instituto Federal do Espírito Santo - IFES \\ zamora@,ifes.edu.br \\ Emerson Wagner Mainardes, Doutor \\ Fucape Business School \\ emerson@fucape.br
}

Recebido em 03/maio/2017

Aprovado em 05/agosto/2018

Sistema de Avaliação: Double Blind Review 


\title{
RESUMO
}

O marketing interno (MI) e a orientação para o mercado interno (OMI) são estratégias que permitem satisfazer as necessidades dos funcionários com o intuito de criar valor para $o$ cliente final. O objetivo do artigo é identificar e comparar as subdimensões da OMI que afetam a satisfação no trabalho de docentes e do pessoal administrativo em uma instituição de ensino pública. Revisou-se a literatura sobre MI e OMI, especialmente dentro do contexto educacional. A pesquisa teve abordagem quantitativa e descritiva. A amostra foi de 624 funcionários que responderam a um questionário baseado no modelo de Gounaris (2006). Os resultados demonstraram que as duas carreiras analisadas possuem visões diferentes da organização ao que se refere à OMI, sendo que apenas uma subdimensão: Comunicação entre gestores e funcionários foi significativa na satisfação do trabalho de ambas carreiras. Porém, para o pessoal administrativo outras três subdimensões: Treinamento, Atenção aos grupos com necessidades comuns e Identificação das necessidades do cliente interno mostraram serem significativas. Em suma, existem variavéis comuns que afetam a satisfação das diferentes carreiras, porém algumas variaveis afetam apenas um grupo.

Palavras chave: Orientação para o Mercado Interno. Satisfação no Trabalho. Instituição educacional. Docente. Pessoal Administrativo.

\begin{abstract}
Internal marketing (IM) and internal market orientation (IMO) are strategies that allow satisfay the needs of employees in order to create value for the final customer. The purpose this article is identify and compare the IMO sub-dimensions that affect teacher and administrative staff satisfaction in a public educational institution. In first moment, the literature on IM and IMO has been revised, especially within the educational context. The research had a quantitative and descriptive approach. The sample was of 624 workers that answered a questionnaire based on the model of Gounaris (2006). The results showed that the two careers analyzed had different views of the IMO in organization, it only one subdimension: Communication between managers and employees was significant in the work of both careers. However for the administrative staff three other sub-dimensions: Training, Attention to groups with common needs and Identification of internal customer needs shown to be significant. In resume concluded that there are common variables that affect the satisfaction of the different careers, but some variables affect only one group.
\end{abstract}

Keywords: Internal Market Orientation. Job Satisfaction. Educational Institution. Teachers. Administrative Staff. 


\section{SATISFAÇÃO NO TRABALHO SOB A PERSPECTIVA DA ORIENTAÇÃO PARA O MERCADO \\ INTERNO DE UMA INSTITUICCÃO DE ENSINO PÚBLICA: DOCENTES X ADMINISTRATIVO \\ DOI: http://dx.doi.org/10.5007/1983-4535.2018v11n3p20}

\section{INTRODUÇÃO}

$\mathrm{Na}$ atual dinâmica do mercado, as instituições educacionais estão expostas a forças competitivas, enfatizando a necessidade de uma melhor relação com o aluno (HEMSLEYBROWN; OPLATKA, 2006). Uma vez que a educação é um serviço intangível e o cliente está envolvido diretamente na entrega do produto, alguns modelos de gestão começaram a ser implementados para melhorar a qualidade dessas organizações (GREEN, 1994).

O marketing interno (MI) é uma das várias estratégias que são utilizadas para melhorar a qualidade dos serviços oferecidos (LINGS, 2004; GOUNARIS, 2008; HUNG, 2012; AMANGALA; AMANGALA, 2013). Esta prática consiste em considerar os funcionários como cliente interno da organização, a partir da premissa que a satisfação de suas necessidades impacta na criação de valor para o cliente externo (BERRY; PARASURAMAN, 1991, AHMED; RAFIQ, 2003).

Para direcionar a aplicação do MI nas organizações surgiu a orientação para o mercado interno (OMI), que orienta o comportamento da empresa de forma a satisfazer as necessidades dos seus funcionários (LINGS, 2004; GOUNARIS, 2010). A mensuração da OMI, utilizada neste estudo, foi a elaborada por Gounaris (2006) que é composta por três dimensões que abrangem a capacidade da organização identificar, disseminar e responder as necessidades dos funcionários para satisfazê-los. Essas dimensões são compostas por dez subdimensões: Identificação das necessidades do cliente interno; Condições do mercado de trabalho; Atenção a grupos com necessidades comuns; Atenção ao Público Interno; Comunicação entre gestores e Funcionários; Comunicação entre gestores; Descrição do Trabalho; Sistema de remuneração; Treinamento; Preocupação da alta direção com os colaboradores.

$\mathrm{Na}$ educação, as instituições educacionais (IE) ainda são um campo de estudo relativamente inexplorado (ALGARINI; TALIB, 2014), tanto na prestação de serviços para o ambiente público, quanto na aplicação de teorias de marketing por essas organizações (HEMSLEY-BROWN; OPLATKA, 2006). Estudos anteriores buscaram entender o que satisfaz os professores por meio do marketing interno (HUNG, 2012; ALTARIFI, 2014; SHABBIR; SALARIA, 2014), enquanto outros usaram a OMI como ferramenta para melhorar os serviços ao aluno (RODRIGUES, CARLOS; 2012) e a comunicação interna nestas organziações (ALGARNI; TALIB, 2014). Pórem, nenhum estudo abordou a organização como um todo, abrindo uma lacuna para mensurar a OMI por grupo de 
funcionários que compõe a instituição de ensino.

Em geral, as organizações são compostas por grupos de funcionários, os quais são segmentados por carreiras ou pelas atividades que desempenham. No caso das instituições de ensino, o grupo de trabalho é formado pelos docentes e o pessoal administrativo, onde ambos contribuem na prestação de serviço ao aluno. Assim, o objetivo deste estudo foi identificar e comparar as subdimensões da OMI que afetam a satisfação no trabalho das duas diferentes carreiras em uma organização de ensino pública.

Como tema acadêmico, este estudo propõe-se investigar empiricamente a OMI em IE, visto que existem poucos estudos com esse propósito (DE FARIAS, 2010; AMANGALA E AMANGALA, 2013). Além disso, diferente dos estudos anteriores em instituições de ensino (RODRIGUES; PINHO, 2012; SHABBIR; SALARIA, 2014) que analisaram apenas um grupo da IE (docentes), este estudo amplia a literatura, por realizar uma pesquisa de OMI comparando a percepção de duas diferentes carreiras (administrativa e docente) em uma mesma instituição de ensino. De acordo com Shabbir e Salaria (2014), as instituições educacionais representam um setor dinâmico com alto grau de interação entre funcionários e clientes. Consequentemente, investigar as carreiras separadamente torna-se um elemento chave para a compreensão de todo o contexto.

A relevância para as organizações, principalmente na área da educação, dá-se pela identificação e comparação das subdimensões associadas à satisfação de ambas as carreiras, fato que permite à organização formular políticas específicas de gestão de pessoas (GOUNARIS, 2008), aumentar o compromisso organizacioanal (LEE et al., 2015) e otimizar a comunicação interna (SHIMA; GEORGE, 2014), de forma a promover a satisfação no trabalho e, conseqüentemente, a satisfação dos alunos (ALTARIFI, 2014).

\section{REFERENCIAL TEÓRICO}

\subsection{MARKETING INTERNO, ORIENTAÇÃO PARA O MERCADO INTERNO E A SATISFAÇÃO NO TRABALHO}

O marketing interno (MI) é uma ferramenta que se utiliza de estratégias e elementos do marketing tradicional. O objetivo é estreitar a relação entre a organização e seus funcionários, de forma a satisfazê-los (RAFIQ; AHMAND, 2000; SHABBIR; SALARIA, 2014). Entende-se que as transações internas entre a organização e seus empregados possuem 
o mesmo valor das interações externas, devido o fato de influenciá-las. Consequentemente, tal perspectiva possibilita a ampliação de melhorias nos níveis de serviço (BERRY, 1987).

No entanto, a operacionalização do MI nas organizações ainda é limitada, apesar de as teorias serem estudadas há algumas décadas (LINGS; GREENLEY, 2005; GOUNARIS, 2010). Por este motivo, surgiu a necessidade de desenvolver um instrumento que avaliasse a amplitude dessa estratégia por direcionar ações internas especifícas que atendessem as necessidade dos funcionários, assim surgiu a OMI (GOUNARIS, 2006).

Com a orientação para o mercado interno (OMI), a organização pode concentrar a sua atenção na identificação e compreensão das necessidades do mercado interno, de forma a desenvovler uma relação de confiança e compromisso entre empregadores e empregados (GOUNARIS, 2006; LINGS; GREENLEY, 2010). Nesse contexto, Lings e Greenley (2005) destacam os funcionários como clientes internos que dependem da organização para satisfazer suas necessidades, e conforme forem satisfeitas, impactará diretamente o serviço que esses funcionários prestarão ao mercado externo.

A base para a formulação da OMI foi desenvolvida a partir do conceito de orientação para o mercado externo (LINGS, 2004). Gounaris (2006), para mensurar a OMI, criou uma abordagem hierárquica (Quadro 1), que consiste em 3 dimensões relacionadas a 10 subdimensões, que permitem gerar informação, disseminar e responder aos empregados (AMANGALA; AMANGALA, 2013).

Quadro 1 Orientação para o mercado interno

\begin{tabular}{|c|l|}
\hline \multicolumn{1}{|c|}{ Dimensões } & \multicolumn{1}{c|}{ Subdimensões } \\
\hline $\begin{array}{c}\text { Geração e conhecimento dos } \\
\text { empregados }\end{array}$ & $\begin{array}{l}\text { S1 - Identificação das necessidades do cliente interno; } \\
\text { S2 - Condiçães do mercado de trabalho; } \\
\text { S3 - Atenção a grupos com necessidades comuns; } \\
\text { S4 - Atenção ao Público Interno }\end{array}$ \\
& \\
\hline Disseminação do conhecimento & S5 - Comunicação entre gestores e funcionários; \\
& S6 - Comunicação entre gestores; \\
\hline Respostas ao conhecimento gerado & $\begin{array}{l}\text { S7 - Descrição do trabalho; } \\
\text { S8 - Sistema de remuneração; } \\
\\
\text { S9 - Treinamento; } \\
\text { S10 - Preocupação da alta direção com os colaboradores }\end{array}$ \\
\hline
\end{tabular}

Fonte: Gounaris (2006)

Nota: Adaptado pelos autores 
A forma encontrada por Gounaris (2006) para quantificar a OMI foi desenvolver e validar um questionário a partir das subdimensões (Quadro 1) que fosse direcionado às organizações que prestam serviço. Várias áreas de serviço já utilizaram o questionário, tais como hotéis (SANCHEZ-HERNANDEZ; MIRANDA, 2011), bancos (TORTOSA; MOLINER; SANCHEZ, 2009), organizações públicas (MAINARDES; CERQUEIRA, 2015) e universidades (CARLOS; RODRIGUES, 2012).

A proposta é avaliar o ambiente interno e verificar a existência de conexão entre o sentimento de satisfação sentido pelos funcionários e a tradução de tais sentimentos e atitudes nos seus serviços ao cliente (GOUNARIS, 2006; AMANGALA; AMANGALA, 2013). Após as informações obtidas e compartilhadas na organização, o empregado percebe que suas necessidades estão sendo consideradas, e contribui com os outros agentes do processo (stakeholders) (LINGS, GREENLEY, 2010; ALGARNI; TALIB, 2014).

A satisfação do funcionário pode ser afetada positivamente conforme ocorre a aplicação da OMI na organização, e como consequência, uma vez os funcionarios satisfeitos contribuirão para satisfação do cliente final.

\subsection{ESTRATÉGIAS DE MARKETING E INSTITUIÇÕES EDUCACIONAIS}

A competitividade, especialmente na contratação de professores e na busca de qualidade de serviço, é um desafio no ambiente das instituições educacionais (IE) (GREEN, 1994). Neste contexto, é necessário que as IE entrem no mercado com maior visibilidade (HEMSLEY-BROWN; OPLATKA, 2010) e se esforcem em alcançar a excelência para suprir as demandas dos estudantes e do mercado (ALGARNI; TALIB, 2014).

No entanto, a satisfação do aluno está intimamente relacionada com a qualidade do serviço oferecido pela IE, que procura formas mais eficazes para melhorar o serviço prestado (YEO, 2008; VOON, 2008). Isso implica a necessidade de aplicar as teorias de marketing que tiveram bons resultados no mundo dos negócios (HEMSLEY-BROWN; OPLATKA, 2010; SHIMA; GEORGE, 2014).

As estratégias de marketing são utilizadas para melhorar o desempenho nas IE. Por exemplo, Algarini e Talib (2014) usaram a Orientação para o Mercado como uma das etapas para entender os stakeholders das IE e concluíram que a satisfação do aluno ocorrerá após a satisfação dos funcionários. A relação entre marketing interno e a satisfação no trabalho com 


\section{SATISFAÇÃO NO TRABALHO SOB A PERSPECTIVA DA ORIENTAÇÃO PARA O MERCADO \\ INTERNO DE UMA INSTITUIÇÃO DE ENSINO PÚBLICA: DOCENTES X ADMINISTRATIVO \\ DOI: http://dx.doi.org/10.5007/1983-4535.2018v11n3p20}

a eficácia escolar também foi confirmada por Hung (2012), que concluiu que a satisfação dos professores está diretamente relacionada com a qualidade da educação oferecida aos alunos.

Ainda utilizando o marketing interno como estratégia, Shabbir e Salaria (2014) e Altarife (2014) comprovaram que a satisfação do aluno também está relacionada à satisfação do professor na área educacional. Uma das razões é o alto nível de interatividade neste tipo de serviço, uma vez que os funcionários (professores e administrativos) mantêm contato direto e regular com os clientes (estudantes) (VOON, 2008; SHABBIR; SALARIA, 2014).

A Orientação para o mercado interno também foi aplicada como ferramenta estratégica em instituições educacionais (CARLOS; RODRIGUES, 2012). A conclusão de Carlos e Rodrigues (2012) foi à existência de uma relação positiva entre o grau de OMI, satisfação no trabalho, compromisso e desempenho dos funcionários em IE. As mesmas relações positivas foram comprovadas por Lee et.al (2015) em outro tipo de organização de serviço.

Como qualquer outra organização, as IE necessitam melhorar a qualidade do serviço oferecido e garantir sua permancência no mercado. Como alternativa, ferramentas com foco no mercado interno, que visem à satisfação dos funcionários, podem ser aplicadas nessas organizações (CARLOS; RODRIGUES, 2012. ALGARNI; TALIB, 2014).

\section{METODOLOGIA}

Dado o objetivo deste estudo, o método utilizado foi quantitativo, descritivo e corte transversal, a fim de identificar e comparar as subsimensões da OMI que afetam a satisfação no trabalho das duas diferentes carreiras em uma instituição de ensino pública.

O campo de estudo foi o Instituto Federal do Espírito Santo (IFES), composto de 20 campi distribuídos no estado, com um total de 2.539 servidores. A escolha deu-se ao fato de o instituto fazer parte de uma rede federal presente em todo o país, que oferece cursos em diversas áreas (exatas, humanas e biológica), desde o nível técnico aos programas de pósgraduação. A amostra foi caracterizada por ser não probabilística por acessibilidade (CRESWEL, 2007).

O instrumento de coleta de dados foi um questionário estruturado, de acordo com o modelo de Gounaris (2006), com 10 subdimensões e 43 afirmações. Vale ressaltar que o questionário original foi desenvolvido com foco no setor privado. Então, foram realizados ajustes para o setor público, conforme proposto pela Mainardes e Cerqueira (2015), sendo retiradas 08 afirmações que não se aplicavam à organização pública. A fim de validar o 
questionário, foi aplicado um pré-teste, que foi enviado a oito funcionários, que não tiveram dificuldade em entender seu conteúdo.

O levantamento final consistiu em 36 afirmações acompanhadas de uma escala de Likert de 5 pontos $(1$ = Discordo totalmente; 5 = Concordo totalmente), sendo construído em duas partes: a primeira consistiu em 1 afirmação relacionada à satisfação no trabalho e por 35 afirmações agrupadas dentro das dimensões e subdimensões. A segunda parte da pesquisa consistiu em questões de perfil dos entrevistados, com relação ao gênero (GEN), idade (ID), carreira (CAR), tempo de serviço (TS), treinamento (FOR) e função de liderança (FCH).

O questionário foi enviado por e-mail a todos os funcionários. A amostra final foi formada por 624 entrevistados (aproximadamente 24,5\% do contingente total de servidores), que foram divididos em dois grupos de acordo com a sua carreira: amostra de pessoal administrativo (1) com 408 entrevistados (65\% da amostra) e amostra de professores (2) com 216 respondentes $(35 \%)$.

No processo de análise de dados, foi realizado primeiramente a caracterização de cada amostra, medida pelas respostas de questões sóciodemográficas. Posteriormente, aplicou-se o teste T com o objetivo de comparar as subdimensões, pois é um método comumente utilizado para avaliar as diferenças entre médias de dois grupos diferentes (HAIR et al., 2005). Com este teste, foi possível identificar as subdimensões da OMI que apresentaram médias com diferenças estatísticas entre o pessoal administrativo e a amostra de docentes.

Em uma segunda etapa, utilizou-se a regressão linear múltipla em cada amostra, cuja variável dependente foi a satisfação com o trabalho e as independentes as 10 subdimensões (Quadro 1). Para compor a nota de cada subdimensão, foi extraída a média por respondente dos indicadores que compõem cada subdimensão.

\section{ANÁLISE E DISCUSSÃO DE DADOS}

\subsection{CARACTERIZAÇÃO DA AMOSTRA}

A amostra foi dividida em dois grupos de acordo com a carreira. A amostra (1) foi formada pelo pessoal administrativo e teve 408 respondentes e a amostra (2) foi formada pelos docentes e teve 216 respondentes.

A amostra do pessoal administrativo foi caracterizada pelo equilíbrio de gênero, com $52 \%$ de mulheres e $48 \%$ de homens. Quanto a formação $43 \%$ possui pós-graduação, e boa parte tem mais de 7 anos na instituição (36\% dos entrevistados). Grande parte dos 
entrevistados dessa amostra não está em posição de liderança, ou seja, 72\% não exerce cargo de chefia.

$\mathrm{Na}$ amostra dos docentes, a maioria dos entrevistados, ou seja, $72 \%$ foi composta por homens, e 51\% deles possuem mestrado. Como na amostra anterior, boa parte dos docentes possuem mais de 7 anos na instituição (39,5\%), e a maioria não ocupa posições de gestão $(76,5 \%)$.

A amostra selecionada mostrou-se válida em todas as subdimensões de controle, exceto pelo fato do pessoal administrativo apresentar um maior volume de respostas, apesar do quadro dos funcionários do instituto ser equilibrado entre carreiras. As demais subdimensões foram semelhantes ao empregado médio da Instituição de Ensino pesquisada: predominantemente homens, pós-graduação, vida funcional entre 7 a 10 anos e a maioria não exerce cargos de gestão.

\subsection{COMPARAÇÃO DAS MÉDIAS DE CADA SUBDIMENSÃO ENTRE A AMOSTRA DAS DUAS CARREIRAS: PESSOAL ADMINISTRATIVO E DOCENTES}

Para comparar as médias de cada subdimensão atribuídas pelas duas carreiras (docente e administrativo), utilizou-se o teste $\mathrm{t}$ de Student. A Tabela 1 apresenta as medidas descritivas dos dados (tamanho de cada amostra, média, desvio padrão e amostra de erro padrão), o teste de Levene para homogeneidade de variâncias e o teste $\mathrm{t}$ comparando essas duas médias.

Após a análise de cada subdimensão, as médias foram estatisticamente diferentes ( $\mathrm{p}$ valor $<0,05$ ) em seis subdimensões dos 10 analisadas, representando $60 \%$ de diferença entre as médias. As subdimensões que mostraram diferenças entre as carreiras foram: Identificação das necessidades do cliente interno (S1); Condições do Mercado de Trabalho (S2); Atenção ao Público Interno (S4); Comunicação entre gestores (S6); Descrição do Trabalho (S7) e Preocupação da alta direção com os colaboradores (S10). Em todos os casos, os professores atribuíram as maiores notas, em comparação com o pessoal administrativo.

A média da subdimensão Identificação das necessidades do cliente interno (S1) apresentou baixo resultado em ambas as amostras. Os docentes $(\mathrm{M}=3,03)$ consideram razoável o esforço da instituição em identificar as necessidades dos empregados e boa parte do pessoal administrativo $(\mathrm{M}=2,83)$ considerou como insatisfatória as ações da IE neste sentido. Na opinião do pessoal administrativo há pouca ação com o objetivo de identificar suas necessidades. Pode-se melhorar essa situação por meio de avaliações regulares e 
pesquisa de mercado interno, onde é possivel mapear as reais necessidades dos funcionários (RODRIGUES; PINHO, 2012).

Tabela 1 Comparação de Médias das Amostras com Valor - $\mathrm{P}<0,05$

\begin{tabular}{|c|c|c|c|c|c|c|}
\hline Subdimensões & CAR & $\mathbf{N}$ & Média & $\begin{array}{l}\text { Desvio } \\
\text { Padrão }\end{array}$ & $\begin{array}{c}\text { Erro } \\
\text { padrão da } \\
\text { média }\end{array}$ & Sig. (2 extremidades) \\
\hline \multirow{2}{*}{ SATISFAÇÃO } & $\mathrm{D}$ & 215 & 3,67 & 1,14 & 0,08 & \multirow{2}{*}{0,08} \\
\hline & $\mathrm{PA}$ & 409 & 3,51 & 1,06 & 0,05 & \\
\hline \multirow{2}{*}{ S1 } & $\mathrm{D}$ & 215 & 3,03 & 0,89 & 0,06 & \multirow{2}{*}{0,01} \\
\hline & PA & 409 & 2,82 & 0,90 & 0,04 & \\
\hline \multirow{2}{*}{$\mathrm{S} 2$} & $\mathrm{D}$ & 215 & 2,82 & 1,05 & 0,07 & \multirow{2}{*}{0,03} \\
\hline & PA & 409 & 2,63 & 1,06 & 0,05 & \\
\hline \multirow{2}{*}{ S3 } & $\mathrm{D}$ & 215 & 2,34 & 0,73 & 0,05 & \multirow{2}{*}{0,16} \\
\hline & $\overline{\mathrm{PA}}$ & 409 & 2,42 & 0,72 & 0,04 & \\
\hline \multirow{2}{*}{ S4 } & $\mathrm{D}$ & 215 & 2,64 & 0,73 & 0,05 & \multirow{2}{*}{0,00} \\
\hline & $\mathrm{PA}$ & 409 & 2,43 & 0,72 & 0,04 & \\
\hline \multirow[b]{2}{*}{ S5 } & $\mathrm{D}$ & 215 & 3,19 & 0,97 & 0,07 & \multirow[t]{2}{*}{0,96} \\
\hline & $\mathrm{PA}$ & 409 & 3,20 & 0,96 & 0,05 & \\
\hline \multirow{2}{*}{ S6 } & $\mathrm{D}$ & 215 & 2,94 & 0,57 & 0,04 & \multirow[t]{2}{*}{0,00} \\
\hline & PA & 409 & 2,78 & 0,64 & 0,03 & \\
\hline \multirow{2}{*}{ S7 } & $\mathrm{D}$ & 215 & 3,30 & 0,82 & 0,06 & \multirow{2}{*}{0,00} \\
\hline & $\overline{\mathrm{PA}}$ & 409 & 3,08 & 0,79 & 0,04 & \\
\hline \multirow{2}{*}{ S8 } & $\mathrm{D}$ & 215 & 2,81 & 1,26 & 0,09 & \multirow{2}{*}{0,23} \\
\hline & $\mathrm{PA}$ & 409 & 2,69 & 1,26 & 0,06 & \\
\hline \multirow{2}{*}{ S9 } & $\mathrm{D}$ & 215 & 2,66 & 0,75 & 0,05 & \multirow[t]{2}{*}{0,89} \\
\hline & PA & 409 & 2,65 & 0,77 & 0,04 & \\
\hline \multirow{2}{*}{$\mathrm{S} 10$} & $\mathrm{D}$ & 215 & 3,01 & 0,85 & 0,06 & \multirow[t]{2}{*}{0,00} \\
\hline & $\mathrm{PA}$ & 409 & 2,81 & 0,75 & 0,04 & \\
\hline
\end{tabular}

Fonte: Dados da Pesquisa

Legenda: D- Docente ; PA - Pessoal Administrativo

S1- Identificação das necessidades do cliente interno; S2- Condições do mercado de trabalho; S3Atenção a grupos com necessidades comuns; S4- Atenção ao Público Interno; S5- Comunicação entre gestores e Funcionários; S6- Comunicação entre gestores; S7- Descrição do Trabalho; S8- Sistema de remuneração; S9- Treinamento; S10- Preocupação da alta direção com os colaboradores. 


\section{SATISFAÇÃO NO TRABALHO SOB A PERSPECTIVA DA ORIENTAÇÃO PARA O MERCADO

Apesar da média atribuída a subdimensão Condições do Mercado de Trabalho (S2) mostrar ser diferente estatisticamente entre as duas amostras, em ambos os casos a média pode ser considerada insatisfatória. Pórem, os docentes $(\mathrm{M}=2,82)$ tiveram média ligeiramente superior ao pessoal administrativo $(\mathrm{M}=2,63)$. Na visão das duas carreiras, a IE se preocupa pouco em conhecer o mercado de trabalho, mesmo sendo um fator importante na OMI para gerar conhecimento sobre os funcionários (GOUNARIS, 2008). O motivo possa ser explicado pelo fato da IE ser pública, e o serviço público ser um mercado com características específicas, que dificulta uma análise mais profunda dessa subdimensão (MAINARDES; CERQUEIRA, 2015).

A subdimensão Atenção ao Público Interno (S4) apresentou médias diferentes entre as amostras, onde a média dos docentes foi de 2,63 e a média do pessoal administrativo de 2,43. Embora os professores excedam em 0,20 a média do pessoal administrativo, ambas as médias indicam serem insatisfatórias. Demonstra a pouca preocupação com as necessidades individuais do funcionário, o que minimiza o sentimento de valor desse funcionário em relação ao seu trabalho. O resultado corroborou com o estudo de Rodrigues e Pinho (2012), que apontou como causa a dificuldade e interesse dos gestores das instituições de ensino em gerar informação sobre os desejos e sentimentos das pessoas individualmente acerca de seus trabalhos.

Outra média estatisticamente diferente foi a subdimensão Comunicação entre gestores (S6). Foi considerada por ambas as amostras como um processo relativamente falho na instituição. Os docentes $(\mathrm{M}=2,93)$ mantiveram uma média superior à do pessoal administrativo $(M=2,77)$. Possivelmente, o fato de que todos os superiores de professores são da mesma carreira pode facilitar o intercâmbio de informações, situação contrária ao pessoal administrativo, em que um professor pode ocupar a liderança no setor administrativo. Esta possibilidade pode gerar dificuldades na relação e prejudicar a comunicação horizontal, condição relevante para as organizações (MONTANA; CHARNOV, 2003).

A subdimensão Descrição do Trabalho (S7) foi outra média que mostrou diferenças entre as amostras. Mais uma vez, os professores relataram uma média $(M=3,3)$ superior aos servidores administrativos $(M=3,08)$, mas ambas as carreiras consideraram de forma regular as atividades que realizam em relação às suas necessidades e objetivos. A diferença poderia ser explicada pelo fato de os docentes terem uma função bem definida, regida por legislação 
específica, enquanto o pessoal administrativo possuir restrições quanto às suas tarefas e, em muitos casos, estas não são bem definidas.

Por fim, a subdimensão Preocupação da alta direção com os colaboradores (S10) apareceu como regular na opinião dos docentes $(M=3,0)$ enquanto que no ponto de vista do pessoal administrativo $(M=2,81)$ foi considerada relativamente baixa. Este resultado aponta que a interação dos gestores é limitada, de acordo com uma parcela dos entrevistados, mantendo uma atitude indiferente às necessidades individuais e coletivas, dificultando que ações pontuais sejam elaboradas e implementadas de forma a satisfazê-los (LINGS; GREENLEY, 2010).

Nas demais subdimensões, não foi encontrada diferenças estatisticamente significativas entre essas duas amostras. A subdimensão dependente "Satisfação no Trabalho" também mostrou homogeneidade entre as carreiras, com satisfação regular. O resultado foi compatível com estudos anteriores no setor público, como Mainardes e Cerqueira (2015) e Rodrigues e Pinho (2012). O resultado é justificado por Rodrigues e Pinho (2012) como falta de sensibilidade dos gestores públicos em relação às necessidades dos funcionários, apontando que estes gestores geralmente não tomam medidas corretivas para minimizar a insatisfação.

Comparar os resultados entre as médias permitiram verificar a pouca linearidade na percepção entre as carreiras de uma mesma organização (LINGS, 2005). Apesar de ambas as carreiras não estarem plenamente satisfeitas com nenhuma das subdimensões, uma vez que nenhuma média alcançada está acima de quatro. Os docentes, ainda, apresentaram médias superiores ao pessoal administrativo em 90\% das subdimensões e médias estatisticamente diferentes em $60 \%$ das subdimensões. Isso comprova as diferentes formas que uma organização pode ser vista pelos grupos de trabalho que a compõe. Dessa forma, não parece ser sensato que a organização considere todos os grupos funcionais de um mesmo modo.

\subsection{REGRESSÃO LINEAR MÚLTIPLA}

Após realizar a análise descritiva das amostras e compará-las (T-Test), foram aplicadas duas regressões lineares múltiplas para identificar as subdimensões da OMI de cada carreira que pode afetar a satisfação dos trabalhadores. 
$\mathrm{Na}$ análise do modelo de regressão dos servidores administrativos (1), conforme mostra a Tabela 2, observa-se que o R2 ajustado indica que a satisfação no trabalho pode ser explicada por $16,8 \%$ das subdimensões. O modelo F é 6,29 e P $<0,05$, significativo a $95 \%$.

Tabela 2 Resumo do Modelo - Servidores Administrativos

\begin{tabular}{|c|c|c|c|c|c|c|c|c|c|c|}
\hline \multirow[b]{2}{*}{ Mod } & \multirow[b]{2}{*}{$\mathrm{R}$} & \multirow[b]{2}{*}{$\mathrm{R}^{2}$} & \multirow{2}{*}{$\begin{array}{c}\mathrm{R}^{2} \\
\text { ajustado }\end{array}$} & \multirow{2}{*}{$\begin{array}{c}\text { Erro } \\
\text { padrão da } \\
\text { estimativa }\end{array}$} & \multicolumn{5}{|c|}{ Estatísticas de mudança } & \multirow[b]{2}{*}{$\begin{array}{l}\text { Durbin- } \\
\text { Watson }\end{array}$} \\
\hline & & & & & $\begin{array}{c}\text { Alteração } \\
\text { de }^{2}\end{array}$ & $\begin{array}{c}\text { Alteração } \\
\text { F }\end{array}$ & $\begin{array}{c}\mathrm{df} \\
1\end{array}$ & $\begin{array}{l}\mathrm{df} \\
2\end{array}$ & $\begin{array}{c}\text { Sig. } \\
\text { Alteração } \\
\text { F }\end{array}$ & \\
\hline 4 & ,420 & $\begin{array}{c}0,1 \\
7\end{array}$ & 0,16 & 0,96 & 0,01 & 6,29 & 1 & $\begin{array}{c}40 \\
4\end{array}$ & 0,01 & 1,86 \\
\hline
\end{tabular}

Método de estimação: Stepwise

Testes de Validez:

ANOVA: significativo

Teste de Aderência Kolmogorov-Smirnov: Suporta hipótese de Aderência a distribuição normal

Teste de Aleatoriedade: Suporta a hipótese de Aleatoriedade

Teste de Homocedasticidade: Suporta a hipótese de homocedasticidade

a. Preditores: (Constante), S5, S9, S3, S1

b. Variável Dependente: SAT

Tabela 3 Coeficientes - Regressão Servidores Administrativos

\begin{tabular}{|c|c|c|c|c|c|c|c|c|c|c|c|c|}
\hline \multirow{2}{*}{ Modelo } & \multicolumn{2}{|c|}{$\begin{array}{l}\text { Coeficientes } \\
\text { não } \\
\text { padronizados }\end{array}$} & \multirow{2}{*}{$\begin{array}{c}\text { Coeficientes } \\
\text { padronizado } \\
\text { s } \\
\text { Beta }\end{array}$} & \multirow{2}{*}{$\mathrm{t}$} & \multirow{2}{*}{ Sig. } & \multicolumn{2}{|c|}{$\begin{array}{c}95,0 \% \\
\text { Intervalo } \\
\text { de } \\
\text { Confiança } \\
\text { para B }\end{array}$} & \multicolumn{3}{|c|}{ Correlações } & \multicolumn{2}{|c|}{$\begin{array}{l}\text { Estatísticas de } \\
\text { colinearidade }\end{array}$} \\
\hline & B & $\begin{array}{l}\text { Erro } \\
\text { Padrão }\end{array}$ & & & & . & 莺 & 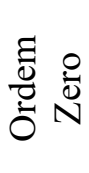 & 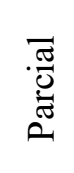 & 苛 & $\begin{array}{l}\frac{\pi}{0} \\
\frac{\pi}{0} \\
\frac{\pi}{0} \\
0\end{array}$ & VIF \\
\hline \begin{tabular}{l|l} 
(Const \\
ante)
\end{tabular} & 1,31 & 0,26 & & 4,94 & 0,00 & $\begin{array}{c}0,7 \\
9\end{array}$ & $\begin{array}{c}1,8 \\
3\end{array}$ & & & & & \\
\hline SD5 & 0,24 & 0,06 & 0,22 & 3,94 & 0,00 & $\begin{array}{c}0,1 \\
2\end{array}$ & $\begin{array}{c}0,3 \\
7\end{array}$ & \begin{tabular}{|c|}
0,3 \\
5
\end{tabular} & $\begin{array}{c}0,1 \\
9\end{array}$ & 0,18 & 0,64 & 1,56 \\
\hline SD9 & 0,17 & 0,07 & 0,12 & 2,30 & 0,02 & $\begin{array}{c}0,0 \\
2\end{array}$ & $\begin{array}{c}0,3 \\
1\end{array}$ & $\begin{array}{c}0,2 \\
7\end{array}$ & $\begin{array}{c}0,1 \\
1\end{array}$ & 0,10 & 0,76 & 1,33 \\
\hline SD3 & 0,20 & 0,07 & 0,13 & 2,95 & 0,00 & $\begin{array}{c}0,0 \\
7\end{array}$ & $\begin{array}{c}0,3 \\
3\end{array}$ & \begin{tabular}{|c|}
0,1 \\
2
\end{tabular} & $\begin{array}{c}0,1 \\
5\end{array}$ & 0,13 & 0,99 & 1,01 \\
\hline SD1 & 0,18 & 0,07 & 0,15 & 2,51 & 0,01 & $\begin{array}{c}0,0 \\
4\end{array}$ & $\begin{array}{c}0,3 \\
2\end{array}$ & $\begin{array}{c}0,3 \\
3\end{array}$ & $\begin{array}{c}0,1 \\
2\end{array}$ & 0,11 & 0,54 & 1,86 \\
\hline
\end{tabular}

Fonte: Dados da Pesquisa

Legenda (Subdimensão): S5 - Comunicação entre gestores e Funcionários; S9- Treinamento; S3 Atenção aos grupos com necessidades comuns; S1- Identificação das necessidades do cliente interno a. Variável Dependente: SAT 


\section{SATISFAÇÃO NO TRABALHO SOB A PERSPECTIVA DA ORIENTAÇÃO PARA O MERCADO

No caso do pessoal administrativo, de acordo com a Tabela 3, das 10 subdimensões propostas por Gounaris (2006), apenas quatro foram significativas para afetar a satisfação com o trabalho: Comunicação entre gestores e funcionários (S5); Treinamento (S9); Atenção a grupos com necessidades comuns (S3) e Identificação das necessidades do cliente interno (S1). As outras subdimensões não foram mencionadas porque não foram significativas para afetar a satisfação no trabalho do grupo em questão.

A subdimensão comunicação entre gestores e funcionários (S5) demonstrou relevância para afetar a satisfação com o trabalho do pessoal administrativo da mesma forma que nos trabalhos de De Nobile e McCormick, (2008), Lings, Greenley (2010), Shima e George (2014) e Kaled e Salem (2013). O compartilhamento de informações entre o chefe e o subordinado é importante na organização, pois permite criar vínculos entre funcionários e gerentes, melhora a compreensão dos objetivos organizacionais pelos funcionários (KELLER et al, 2006; ALTARIFI, 2014) e afeta a satisfação com seu trabalho.

A subdimensão Treinamento (S9) foi significativa para afetar a satisfação do pessoal administrativo. O resultado indica que o pessoal adminstrativo poderá ter satisfação em seu trabalho se estiver preparado para desenvolvê-lo. O treinamento torna-se ainda mais necessário e eficaz quando se considera que a IE, para manter um serviço de qualidade, tem de atender às necessidades das empresas locais, bem como se adaptar à mudança do aluno (YEO, 2008).

Outra subdimensão que afeta a satisfação do pessoal administrativo é Atenção a grupos com necessidades comuns (S3). A satisfação desses funcionários está relacionada ao modo como a instituição compreende os diferentes grupos e suas necessidades. Trata-se de reconhecer que os segmentos internos possuem características diferentes e necessitam do desenvolvimento de ações específicas e não generalistas (LINGS, 2005; GOUNARIS, 2008).

A última subdimensão significativa para os administrativos foi a identificação das necessidades do cliente interno (S1). Se o objetivo é satisfazer os colaboradores, os gerentes precisam realizar pesquisas internas para gerar informações sobre o público interno, permitindo que sejam atendidas (RODRIGUES; PINHO, 2012). A satisfação no trabalho é mais provável de ocorrer quando o diretor ou a gerência está disposta a interagir com os funcionários da organização (DE NOBILE; MCCORMICK, 2008).

Os dados mostraram que $40 \%$ das subdimensões foram relevantes para explicar a satisfação do pessoal administrativo. Em resumo, para atingir a satisfação desse grupo, a IE 
precisa investir em treinamento direcionado ao trabalho realizado por eles, bem como melhorar a comunicação entre os chefes imediatos e a equipe, considerar os grupos de modo específico e identificar as necesidades dos funcionários.

O modelo de regressão da amostra dos Docentes (2), conforme Tabela 4, indicou um $\mathrm{R}^{2}$ ajustado de 0,126 , o que significa que o modelo explica $12,6 \%$ de relação com a satisfação no trabalho dos professores. O modelo tem $\mathrm{F}$ de 31,77 e $\mathrm{P}<0,05$, significativo em $95 \%$.

Tabela 4 Resumo Modelo - Docentes

\begin{tabular}{|c|c|c|c|c|c|c|c|c|c|}
\hline \multirow[b]{2}{*}{$\mathrm{R}$} & \multirow[b]{2}{*}{$\mathrm{R}^{2}$} & \multirow[b]{2}{*}{$\begin{array}{c}\mathrm{R}^{2} \\
\text { ajustado }\end{array}$} & \multirow{2}{*}{$\begin{array}{l}\text { Erro } \\
\text { padrão da } \\
\text { estimativa }\end{array}$} & \multicolumn{5}{|c|}{ Estatísticas de mudança } & \multirow[b]{2}{*}{$\begin{array}{l}\text { Durbin- } \\
\text { Watson }\end{array}$} \\
\hline & & & & $\begin{array}{c}\text { Alteração } \\
\text { de } \mathrm{R}^{2}\end{array}$ & $\begin{array}{c}\text { Alteração } \\
\text { F }\end{array}$ & df1 & $\mathrm{df} 2$ & $\begin{array}{c}\text { Sig. } \\
\text { Alteração F }\end{array}$ & \\
\hline $360^{\mathrm{a}}$ & 0,13 & 0,12 & 1,06 & 0,13 & 31,77 & 1 & 213 & 0,00 & 1,93 \\
\hline
\end{tabular}

Método de estimação: Stepwise

Testes de Validez:

ANOVA: significativo

Teste de Aderência Kolmogorov-Smirnov: Suporta hipótese de aderência à distribuição normal

Teste de Aleatoriedade: Suporta a hipótese de aleatoriedade

Teste de Homocedasticidade: Suporta a hipótese de homocedasticidade

a. Preditores: (Constante), S5

b. Variável dependente: SAT

A amostra dos docentes teve como significativa somente 1 das 10 subdimensões testadas deste modelo. Como pode ser observado na Tabela 5, apenas a Comunicação entre gestores e funcionarios (S5) foi significativa para afetar a satisfação dessa carreira.

Tabela 5 Coeficientes - Regressão Docentes

\begin{tabular}{|c|c|c|c|c|c|c|c|c|c|c|c|c|}
\hline \multirow[b]{2}{*}{ Modelo } & \multicolumn{2}{|c|}{$\begin{array}{c}\text { Coeficientes } \\
\text { não } \\
\text { padronizados }\end{array}$} & \multirow{2}{*}{$\begin{array}{c}\text { Coeficientes } \\
\text { padronizados } \\
\text { Beta }\end{array}$} & \multirow[b]{2}{*}{$\mathrm{t}$} & \multirow[b]{2}{*}{ Sig. } & \multicolumn{2}{|c|}{$\begin{array}{c}95,0 \% \\
\text { Intervalo de } \\
\text { Confiança } \\
\text { para B }\end{array}$} & \multicolumn{3}{|c|}{ Correlações } & \multicolumn{2}{|c|}{$\begin{array}{l}\text { Estatísticas d } \\
\text { colinearidade }\end{array}$} \\
\hline & B & $\begin{array}{l}\text { Erro } \\
\text { Padrão }\end{array}$ & & & & 莺高离 & 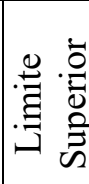 & 节 & 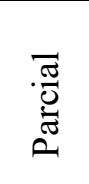 & 玆 & $\begin{array}{l}\frac{\pi}{0} \\
\frac{\pi}{\pi} \\
\frac{\pi}{0} \\
0 \\
\qquad\end{array}$ & VIF \\
\hline $\begin{array}{l}\text { (Cons } \\
1 \text { tante }) \\
\end{array}$ & 2,32 & 0,24 & & 9,33 & 0,00 & 1,83 & 2,81 & & & & & \\
\hline SD5 & 0,42 & 0,07 & 0,360 & 5,63 & 0,00 & 0,27 & 0,56 & 0,36 & 0,36 & 0,36 & 1,0 & 1,0 \\
\hline
\end{tabular}

Legenda (Subdimensão): S5 - Comunicação entre gestores e Funcionários

a. Variável Dependente: SAT

Fonte: Dados da Pesquisa 


\section{SATISFAÇÃO NO TRABALHO SOB A PERSPECTIVA DA ORIENTAÇÃO PARA O MERCADO

Somente a subdimensão Comunicação entre gestores e funcionários (S5) foi significativa para associação com a satisfação dos professores, confirmando estudos anteriores (DE NOBILE; MCCORMICK, 2008; SHABBIR; SALARIA, 2014), que também encontraram uma relação positiva entre comunicação e satisfação entre professores de uma IE. Essa subdimensão também foi abordada por Rodrigues e Carlos (2012) em seu estudo em uma IE, e destacaram a necessidade de investimentos dessas organizações na promoção de uma boa comunicação entre os gestores. Nessa perspectiva, por meio da troca de informações, é possível esclarecer o que se espera dos empregados e o resultado que podem conseguir do trabalho prestado.

As outras subdimensões não se mostraram ser significativas para afetar a satisfação deste grupo. Somente uma subdimensão foi significativa para o grupo de professores, mas podem existir outras subdimensões que não foram consideradas no modelo em questão e que explicam a satisfação no trabalho, como exemplo, a participação dos professores na gestão da IE (OLASKOAGA-LARRAURI et al, 2015).

Ao comparar os resultados da regressão do pessoal administrativo com os resultados da regressão dos docentes, verificou-se que a partir das 10 subdimensões da OMI, apenas uma subdimensão, ou seja, a comunicação entre gestores e funcionários (S5), foi significativa para afetar a satisfação no trabalho das duas carreiras. Ainda, esta subdimensão não apresentou médias diferentes segundo análise descritiva e o Teste- $\mathrm{T}$ aplicado nas amostras. $\mathrm{O}$ resultado mostra que, se houver uma comunicação aberta entre os funcionários e seus superiores em uma IE, é mais provável que tanto docentes quanto o pessoal administrativo mostrem maior satisfação no trabalho (DE NOBILE; MCCORMICK, 2008)

A comunicação tem sido vista como uma importante subdimensão relacionada ao marketing interno e à orientação para o mercado interno em instituições educacionais (RODRIGUES, CARLOS, 2012; SHIMA; GEORGE, 2014; SHABBIR; SALARIA, 2014). De acordo com De Nobile e McCormick (2008), se um professor ou funcionário administrativo perceber que o superior está disposto a ouvi-lo sobre os problemas com o trabalho, ele provavelmente terá atitudes positivas com o seu trabalho.

Por fim, é relevante que as instituições de ensino entendam as necessidades dos funcionários para melhorar seu nível de satisfação e como consequência o serviço prestado ao aluno (ALTARIFE, 2014). Neste estudo, a comunicação, especialmente entre o gestor imediato e o funcionário, indicou ser relevante em ambos os grupos analisados. Assim, uma 
política que vise melhorar a comunicação vertical pode ser aplicada em toda organização e contribuir para satisfação do cliente interno e externo.

\section{CONCLUSÕES}

O objetivo deste estudo foi identificar e comparar as subdimensões da OMI que afetam a satisfação no trabalho das duas diferentes carreiras de em uma organização de ensino pública. Verificou-se que das 10 subdimensões que compõem a OMI, propostas por Gounaris (2006), apenas a Comunicação entre gestores e funcionários impacta na satisfação de ambas as carreiras. Ainda, na amostra do pessoal administrativo (1), outras três subdimensões se mostraram relevantes: Treinamento, Atenção aos grupos com necessidades comuns e Identificação das necessidades do cliente interno.

Os resultados ainda indicam baixa prática de OMI e pouca linearidade de percepção entre as carreiras na instituição de ensino, pois a média das subdimenssões permaneceram entre 2 e 3. Analisar a organização por carreira ou por grupos de trabalho pode ser fundamental para compreensão do todo. Os resultados permitiram concluir que existem variáveis comuns que afetam a satisfação das diferentes carreiras, porém outras variáveis podem ser significativas apenas para um grupo, conforme percebido neste estudo. Como observado, as estratégias de marketing e a satisfação no trabalho apresentaram relação positiva com a eficiência das instituições educacionais (HUNG, 2012). Dessa forma, é apropriado que a instituição formule ações direcionadas para cada grupo, baseadas nas subdimensões encontradas como significativas no estudo, a fim de melhorar o atendimento prestado ao aluno.

Esta pesquisa permitiu a ampliação da literatura na área de marketing interno e OMI nas instituições de ensino. Ainda, como contribuição acadêmica o estudo considerou as duas carreiras distintas em uma IE, visto que os estudos anteriores (HEMSLEY-BROWN; OPLATKA, 2010; CARLOS; RODRIGUES, 2012; ALGARNI; TALIB, 2014; SHABBIR; SALARIA, 2014) limitaram-se a pesquisar apenas o corpo docente das IEs. Além disso, ao compreender a OMI em organizações educacionais, pode-se desenvolver o conceito em outros tipos de organizações (AHMED; RAFIQ, 2003).

A contribuição prática deu-se pela realização da pesquisa de OMI em uma instituição de ensino, reforçando a perspectiva de que algumas subdimensões estão associadas à satisfação no trabalho nesse tipo de organização (CARLOS; RODRIGUES, 2012) e mostrar 
que pode ser aplicada a outras instituições com este perfil. Além disso, os resultados mostram que, dentro da mesma organização, há subdimensões semelhantes que afetam a satisfação no trabalho dos empregados, mas também há diferenças quando analisadas por grupo funcional. A compreensão desta realidade pode favorecer a gestão das instituições de ensino, que poderão criar políticas adequadas para cada grupo, assim como outras organizações que possuem diferentes grupos funcionais trabalhando em conjunto.

Uma das principais limitações do estudo foi que o modelo explica apenas $16,8 \%$ da satisfação dos servidores administrativos e 12,6 \% dos docentes. Assim, nota-se que existem outras variáveis que afetam a satisfação dos funcionários e não foram contempladas na pesquisa, o que enseja um aprofundamento nas pesquisas teóricas e empíricas das IEs e organizações públicas. Outra limitação foi a diferença na amostra entre as carreiras analisadas, sendo a amostra de docente inferior a amostra do pessoal administrativo. Também foi limitador o questionário original ter sido desenvolvido com base em organizações privadas, não compreendendo as especificidades das instituições públicas. Assim, um questionário especifico para o contexto de organizações públicas pode ser desenvolvido e testado.

Para futuros estudos, recomenda-se a continuidade na investigação da OMI em Instituições de Ensino, com intuito de verificar a relação das subdimensões com a satisfação e comprometimento no trabalho dos funcionários. Também sugere realizar uma pesquisa qualitativa com o propósito de se identificar a existência de outras possíveis variáveis relacionadas à OMI que afetam especificamente a satisfação das diferentes carreiras.

\section{REFERÊNCIAS}

AHMED, P. K.; RAFIQ, M. Internal marketing issues and challenges. European Journal of marketing, v. 37, n. 9, p. 1177-1186, 2003. Disponível em $<$ http://dx.doi.org/10.1108/03090560310498813> Acesso em 12 nov .de 2016.

ALGARni, A. M.; TALIB, N.A. A Framework of Measuring the impact of Market Orientation on the outcome of Higher Education Institutions mediated by innovation. International Review of Management and Business Research, v. 3, n. 2, p. 607, 2014. Disponível em < http://www.irmbrjournal.com/papers/1399181158.pdf >. Acesso em 10 nov. de 2016.

ALTARIFI, S. Internal Marketing Activities in Higher Education.International. Journal of Business and Management, v. 9, n. 6, p. p126, 2014. Disponível em $<$ http://www.ccsenet.org/journal/index.php/ijbm/article/view/35274/21204>. Acesso em 15 nov .de 2016. 
AMANGALA, T.; AMANGALA E. Effects of Internal Marketing and Its Components on Organisational Level Customer Orientation: An Empirical Analysis.European Journal of Business and Management, v. 5, n. 8, p. 64-73, 2013. Disponível em < http://iiste.org/Journals/index.php/EJBM/article/view/5097/5186>. Acesso em $10 \mathrm{dez}$.de 2016.

BERRY, L. Big Ideas in Services Marketing. Journal of Services Marketing Vol. 1 No.1, pp.5-9, 1987. Disponível em < $<$ http://dx.doi.org/10.1108/eb008162> Acesso em 08 nov.de 2016.

DE FARIAS, S. A. Internal Marketing (IM): a literature review and research propositions for service excellence. Brazilian Business Review, v. 7, n. 2, p. 99-115, 2010. Disponivel em $<$ http://www.redalyc.org/articulo.oa?id=123021648006 $>$. Acesso em 02 dez .de 2016.

DE NOBILE, J. J.; MCCORMICK, J. Organizational communication and job satisfaction in Australian Catholic primary schools. Educational Management Administration \& Leadership, v. 36, n. 1, p. 101-122, 2008. Disponivel em < http://journals.sagepub.com/doi/pdf/10.1177/1741143207084063 > Acesso em 15 nov. de 2016.

GOUNARIS, S . Internal-market orientation and its measurement. Journal of business research, v. 59, n. 4, p. 432-448, 2006. Disponivel em

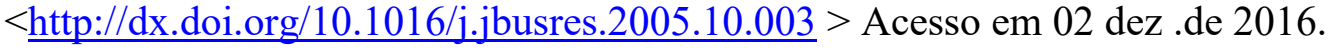

. The notion of internal market orientation and employee job satisfaction: some preliminary evidence. Journal of Services Marketing, v. 22, n. 1, p. 68-90, 2008. Disponivel em $<$ http://dx.doi.org/10.1108/08876040810851978 $>$ Acesso em 10 dez.de 2016.

Internal-market orientation: a misconceived aspect of marketing theory. European Journal of Marketing, vol. 44, pp. 44-60, 2010. Disponível em: http://dx.doi.org/10.1108/03090561011079837>

GOUNARIS, S.; VASSILIKOPOULOU, A.; CHATZIPANAGIOTOU, K.C. Internalmarket orientation: a misconceived aspect of marketing theory. European Journal of Marketing, v. 44, n. 11/12, p. 1667-1699, 2010. Disponivel em < http://dx.doi.org/10.1108/03090561011079837> Acesso em 02 dez .de 2016

GREEN, D. What Is Quality in Higher Education?. Taylor \& Francis, 1900 Frost Road, Bristol, PA 19007-1598, 1994. Disponivel em < https://eric.ed.gov/?id=ED415723 $>$.

KELLER, S. B. et.al, The impact of internal marketing efforts in distribution service operations. Journal of business logistics, v. 27, n. 1, p. 109-137, 2006. Disponivel em < http://onlinelibrary.wiley.com/doi/10.1002/j.2158-1592.2006.tb00243.x/epdf $>$ Acesso em 10 nov. de 2016.

HAIR, J. F. et.al. Fundamentos de métodos de pesquisa em administração. Porto Alegre: Bookman, 2005. 
HEMSLEY-BROWN, J.; OPLATKA, I. Universities in a competitive global marketplace: A systematic review of the literature on higher education marketing. International Journal of public sector management, v. 19, n. 4, p. 316-338, 2006. Disponivel em :

https://pdfs.semanticscholar.org/42c5/67663677adc921a0c89442122e1c84870609.pdf.

Acesso em 10 nov .de 2016.

. Market orientation in universities: A comparative study of two national

higher education systems. International Journal of Educational Management, v. 24, n. 3, p. 204-220, 2010. Disponível em: $\leq$ https://eric.ed.gov/?id=EJ923131>. Acesso em 12 nov. de 2016.

HUNG, C. Internal marketing, teacher job satisfaction, and effectiveness of central Taiwan primary schools. Social Behavior and Personality: an international journal, v. 40, n. 9, p. 1435-1450, 2012. Disponível em:

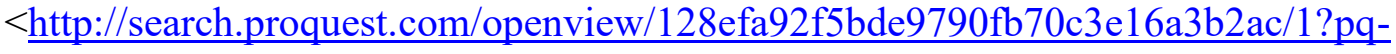
origsite $=$ gscholar \&cbl $=47863>$. Acesso em 02 dez. de 2016.

LEE, W.; CHEN, C.; LEE, C. The relationship between internal marketing orientation, employee commitment, charismatic leadership and performance. In: Proceedings of the 17th International Conference on Electronic Commerce 2015. . Disponível em: < http://dl.acm.org/citation.cfm?id=2781563>. Acesso em 12 nov. de 2016.

LINGS, I. N. Internal market orientation: Construct and consequences. Journal of Business Research, v. 57, n. 4, p. 405-413, 2004. Disponível em: <

http://eprints.qut.edu.au/31775/1/31775.pdf>. Acesso em 10 nov. de 2016.

LINGS, I. N.; GREENLEY, G. E. Measuring internal market orientation. Journal of Service Research, v. 7, n. 3, p. 290-305, 2005. Disponível em $<$ http://jsr.sagepub.com/content/7/3/290.full.pdf+html > Acesso em 10 nov. de 2016.

Internal market orientation and market-oriented behaviours. Journal of Service Management, v. 21, n. 3, p. 321-343, 2010. Disponível em < doi:10.1016/S01482963(02)00274-6> Acesso em 15 nov. de 2016.

MAINARDES, E. W.; CERQUEIRA, A. S. Measuring the internal-market orientation in the public sector. Public Organization Review, p. 1-19, 2015.. Disponivel em < http://hrcak.srce.hr/ojs/index.php/ekonomski-vjesnik/article/view/3093/1840> Acesso em 02 dez. de 2016.

OLASKOAGA-LARRAURI, J. et al. Why has there been a decrease in the job satisfaction of faculty at Spanish universities?. Tertiary Education and Management, v. 21, n. 4, p. 293305, 2015. Disponível em < http://www.tandfonline.com/doi/abs/10.1080/13583883.2015.1068839>. Acesso em 10 nov. de 2016.

RAFIQ, M.; AHMED, P. K. The scope of internal marketing: defining the boundary between marketing and human resource management. Journal of Marketing Management, v. 9, n. 3, p. 219-232, 1993. Disponível em 
$<\underline{\text { http://www.tandfonline.com/doi/pdf/10.1080/0267257X.1993.9964234\#.Ve8XkRFViko }>}$ Acesso em 08 nov. de 2016.

RODRIGUES, A. P.; CARLOS M. J. Market orientation, job satisfaction, commitment and organisational performance: the specific case of local public sector. Transforming Government: People, Process and Policy, v. 4, n. 2, p. 172-192, 2010. Disponível em: $\leq$ http://www.emeraldinsight.com/doi/abs/10.1108/17506161011047398>. Acesso em 10 nov. de 2016.

RODRIGUES, A. P; CARLOS P. J. The impact of internal and external market orientation on performance in local public organisations. Marketing Intelligence \& Planning, v. 30, n. 3, p. 284-306, 2012. Disponível em: <

http://www.isihome.ir/freearticle/ISIHome.ir-22013.pdf>. Acesso em 12 nov. de 2016.

SANCHEZ-HERNANDEZ, M. I.; MIRANDA, F. J. Linking internal market orientation and new service performance. European Journal of Innovation Management, v. 14, n. 2, p. 207-226, 2011. Acesso em $02 \mathrm{dez}$.de 2016.

SHABBIR, M. J.; SALARIA, R. A. Impact of Internal Marketing on Employee Job Satisfaction: An Investigation of Higher Education Institutes of Pakistan. Journal of Marketing Management, v. 2, n. 2, p. 239-253, 2014. Disponível em: < http://jmmnet.com/journals/jmm/Vol_2_No_2_June_2014/14.pdf $>$. Acesso em 10 nov.de 2016.

SHIMA, B.; GEORGE, B. Strategies for the Development of Internal Marketing Orientation in the Private High Education Institutions in Albania. Academic Journal of Interdisciplinary Studies, v. 3, n. 3, p. 393, 2014. Disponível em:

$<$ http://citeseerx.ist.psu.edu/viewdoc/download?doi=10.1.1.665.2761\&rep=rep1\&type $=$ pdf $\#$ p $\underline{\text { age }=393}>$. Acesso em 12 nov .de 2016.

TORTOSA, V.; MOLINER, M. A.; SÁNCHEZ, J. Internal market orientation and its influence on organisational performance. European Journal of Marketing, v. 43, n. 11/12, p. 1435-1456, 2009. Disponível em: $<$ Internal market orientation and its influence on organisational performance>. Acesso em $02 \mathrm{dez}$.de 2016.

VOON, B. H. Servmo: A measure for service-driven market orientation in higher education. Journal of Marketing for Higher Education, v. 17, n. 2, p. 216-237, 2008. Disponível em: $<$ https://ideas.repec.org/a/taf/jmkthe/v17y2008i2p216-237.html $>$. Acesso em 08 nov. de 2016.

YEO, R.K. Brewing service qualityy in higher education: Characteristics of ingredients that make up the recipe. Quality assurance in education, v. 16, n. 3, p. 266-286, 2008. Disponível em: $<$ http:/www.emeraldinsight.com/doi/abs/10.1108/09684880810886277>. Acesso em 12 nov. de 2016. 